\section{Changes in Gluconasturtiin Concentration in Chinese Cabbage with Increasing Cabbage Looper Density}

Fernando A. De Villena

Department of Horticultural Science, University of Minnesota, 1970 Folwell Avenue, St. Paul, MN 55108

Vincent A. Fritz ${ }^{1}$

Department of Horticultural Science, University of Minnesota, 1970 Folwell Avenue, St. Paul, MN 55108; and the Southern Research and Outreach Center, University of Minnesota, 35838 120th Street, Waseca, MN 56093

Jerry D. Cohen

Department of Horticultural Science, University of Minnesota, 1970 Folwell Avenue, St. Paul, MN 55108

William D. Hutchison

Department of Entomology, University of Minnesota, 1980 Folwell Avenue, St. Paul, MN 55108

Additional index words. glucosinolate, herbivory, insect feeding, stress-induced

\begin{abstract}
Changes in the levels of gluconasturtiin (2-phenylethyl glucosinolate), an aromatic glucosinolate, was used to evaluate the response of 'Green Rocket' Chinese cabbage (Brassica campestris ssp. pekinensis $L$.) to the feeding of three and five cabbage looper (Trichoplusia ni Hübner) larvae per plant. Plants were harvested 0, 10, and 17 days after infestation. The change in gluconasturtiin concentration resulting from decreased light capture from diminished leaf area was also studied. All samples were assayed for gluconasturtiin concentration using high-performance liquid chromatography. The gluconasturtiin concentration of plants subjected to five larvae per plant showed a $59 \%$ increase 10 days after infestation compared with noninfested plants. Difference in gluconasturtiin concentration between three and five larvae per plant was nonsignificant. Seventeen days after initial infestation and 7 days after larvae were removed (final harvest), gluconasturtiin concentration did not decrease compared with the previous harvest. Reduced light or leaf area removal did not significantly affect gluconasturtiin concentration.
\end{abstract}

In recent years, the consumption of cruciferous vegetables with increased recognition of their nutraceutical properties has become very important because of the associated benefit of reducing the incidence of cancer and other chronic diseases (Talalay and Fahey, 2001; Wargovich, 2000). A group of secondary compounds, called glucosinolates, is widely present in crucifers. On cellular damage or disruption, hydrolysis of glucosinolates, through the enzyme myrosinase, produces isothiocyanates, which may, in part, account for the chemopreventive properties associated with these vegetables.

Received for publication 20 Feb. 2007. Accepted for publication 21 May 2007.

We thank Mr. Greg Holden and Ms. Lynette Wong for their technical assistance with high-performance liquid chromatography analysis.

Mention of a trademark, proprietary product, or vendor does not constitute a guarantee or warranty of the product by the University of Minnesota and does not imply its approval to the exclusion of other products or vendors that also may be suitable.

${ }^{1}$ To whom reprint requests should be addressed; e-mailvafritz@umn.edu.
The hydrolysis of the aromatic glucosinolate gluconasturtiin releases phenethyl isothiocyanate (PEITC). Isothiocyanates are known to induce phase II detoxification enzymes (Wattenberg, 1990). Previous studies have shown that PEITC provides significant chemoprevention, especially against human prostate cancer (Powolny et al., 2003; Wargovich, 2000; Xiao et al., 2003). Gluconasturtiin is present in several crucifers such as watercress (Nasturtium officinale L.) and Chinese cabbage (Brassica campestris ssp. pekinensis L.). Because of the chemopreventive properties of PEITC, it is desirable to characterize gluconasturtiin (precursor of PEITC) concentration and explore the potential of plants to produce increased amounts of this compound.

The variation in total and individual glucosinolate concentration in a plant is the result of several factors, both abiotic and biotic (Rosa et al., 1997; Smith et al., 2003). Research has also shown that glucosinolate levels are not constant throughout the phenology of a plant, particularly in early growth stages. Young photosynthetically active tissues are believed to be the major sites of synthesis or storage of indole glucosinolates (Chong and Bible, 1974; Ju et al., 1980).

The cabbage looper (Trichoplusia ni Hübner) is a generalist insect native to the United States. It has been extensively used in controlled feeding trials and it was chosen for the present study because of its voracity, lower susceptibility to pesticides, and especially because cabbage plants have been shown to tolerate moderate defoliation before a significant reduction in plant weight occurs (Andaloro and Shelton, 1981). Changes in leaf nutrients incited by herbivory depend on the timing and intensity of feeding (Rostás et al., 2002). However, little has been done to correlate the concentration of glucosinolates with the level of insect pressure.

The possibility of manipulating the metabolism of cruciferous plants to modify its glucosinolate content has generated considerable interest. The potential impact this will have on both consumers and producers will depend on the plant's ability to offer improved nutritional quality, less susceptibility to herbivores/pathogens, increased therapeutic properties, and desirable agronomic, storage, and sensory characteristics (Rosa et al., 1997).

Our objectives were to: 1) determine if there was an effect of cabbage looper larvae feeding on gluconasturtiin concentration in Chinese cabbage by correlating it with the insect pressure imposed on the plant; 2) evaluate the effect after the larvae were removed; and 3) determine if reduced light conditions had an effect on gluconasturtiin concentration.

\section{Materials and Methods}

Two greenhouse experiments were conducted to evaluate the effect of cabbage looper larval feeding on gluconasturtiin concentration in 'Green Rocket' Chinese cabbage plants. This cultivar was used to model the response of cruciferous vegetables to insect herbivory because of its relatively high gluconasturtiin concentration compared with other cultivars (V.A. Fritz, personal communication). A preliminary study was conducted in 2002 to determine the effect of larval feeding of two densities of cabbage looper on gluconasturtiin concentration. A second experiment was designed to evaluate the level of gluconasturtiin over time and was conducted twice in 2003. Greenhouse conditions were maintained constant throughout all experiments $\left(20 \pm 2{ }^{\circ} \mathrm{C}\right.$ with natural photoperiod at $45^{\circ}$ latitude).

Effect of cabbage looper density. On 15 Oct. 2002, two seeds of 'Green Rocket' Chinese cabbage (American Takii, Salinas, CA) were planted $2.5 \mathrm{~cm}$ deep in each cell of a $53 \times 27-\mathrm{cm}$ germination tray (Dillen Products, Middlefield, $\mathrm{OH}$ ) filled with Metro-Mix 200 potting mix $(60 \%$ horticultural vermiculite, $40 \%$ Canadian sphagnum peatmoss, horticultural perlite, and washed sand; Scotts Co., Marysville, $\mathrm{OH}$ ) and watered as needed. On germination, plants were thinned to one per cell. After thinning, $100 \mathrm{mg} \cdot \mathrm{L}^{-1}$ of a 
20N-8.7P-16.6K soluble fertilizer (JR Peters, Allentown, PA) was used to fertilize plants twice a week for the first 2 weeks. From the third week after thinning until the end of the experiment, plants received a weekly application of $200 \mathrm{mg} \cdot \mathrm{L}^{-1}$ of the same $20 \mathrm{~N}-8.7 \mathrm{P}$ $16.6 \mathrm{~K}$ fertilizer. Three weeks after sowing, plants were transplanted to greenhouse benches, filled with a mixture of 1 sterilized sandy loam soil:3 SB 300 (55\% bark, 45\% horticultural vermiculite, Canadian sphagnum peatmoss, horticultural perlite, dolomitic limestone, and gypsum; Sungro Horticulture Canada Ltd., Vancouver, BC) by volume.

The statistical model used was a split-plot design with four replicates. Half the plants in each replicate were covered [photosynthetic photon flux $(P P F): 240 \mu \mathrm{mols} \cdot \mathrm{m}^{-2} \cdot \mathrm{s}^{-1}$ ] with antiaphid netting (Kaplan-Simon Co., Braintree, MA) to prevent unwanted herbivory. This netting would also prevent unwanted predation on the test insects. The remaining half of the plants in each replicate were not covered (PPF: $400 \mu$ mols $\left.\cdot \mathrm{m}^{-2} \cdot \mathrm{s}^{-1}\right)$. The difference in whether the plants were covered with the netting comprised the main plots. No unwanted herbivory was observed in any of the control plants. Larval densities comprised the subplots and were arranged as follows: 1 ) nontreated plants (control), 2) plants infested with three larvae, and 3) plants infested with five larvae. Larval densities were chosen based on previous observations and the need to achieve significant levels of feeding injury while preventing total defoliation. These three larval density treatments were randomly assigned within each block. Each treatment was comprised of four plants per replicate located at each corner of a $38 \times$ 23-cm rectangle, which ran perpendicular to the length of the block. Treatments were separated from each other by four nontreated plants arranged in a similar manner.

On arrival in the laboratory, cabbage looper eggs (USDA-ARS Western Cotton Research Laboratory, Phoenix, AZ) were placed on a plastic tray in a growth chamber for $3 \mathrm{~d}$ at $30{ }^{\circ} \mathrm{C}$ (Environmental Growth Chambers, Chagrin Falls, $\mathrm{OH}$ ). Once the eggs hatched, first-instar larvae were placed on the adaxial surface of the leaves of 4week-old plants using a small, moist paint brush (length, $23 \mathrm{~cm}$ ). One larva was placed per leaf so that each was feeding initially on a separate leaf. Larvae were left on the plant for a period of $10 \mathrm{~d}$, after which two plants per treatment in each replicate were harvested and processed. Before processing, leaf area measurements were taken in plants from both experiments using a belt-drive leaf area meter (Delta-T Devices, Cambridge, UK), so it could be correlated to gluconasturtiin concentration.

Change in gluconasturtiin concentration after herbivory. Seeds of 'Green Rocket' Chinese cabbage were sown on 9 Sept. 2003 in the same way as described previously. They were transplanted 3 weeks after sowing and infested with larvae $7 \mathrm{~d}$ later. Plant spacing, fertilization, infestation, and other cultural practices were conducted as described. The statistical model used was a split-plot design with five replicates with netting treatment being the main plots and larval densities comprising the subplots. Each treatment was comprised of six plants per replicate located at each corner of a $76 \times$ $56-\mathrm{cm}$ rectangle with three plants equally distributed on the longer edge of the rectangle. Treatments were separated from each other by six nontreated plants arranged in a similar manner. Three sequential harvests were conducted and gluconasturtiin concentration at each of them was determined to observe temporal distribution. The first harvest was performed on 12 Oct. 2003 before infestation and the second harvest was conducted $10 \mathrm{~d}$ after infestation. On this date, all larvae were manually removed from all plants. Finally, the third harvest was performed $17 \mathrm{~d}$ after infestation. At each harvest, two plants were harvested from each treatment per replicate, and the roots were discarded. Before processing, leaf area measurements were recorded as described. The experiment was repeated.

Protocol for analysis of glucosinolates. Samples from all experiments were processed and analyzed using the described procedures. First, the weight of Chinese cabbage plants was recorded before washing with deionized water. A 1:15 (w:v) ratio between Chinese cabbage fresh weight and deionized water was used. Water was brought to a boil in a $400-\mathrm{mL}$ heat-resistant beaker covered with aluminum foil. Boiling was conducted to heat-deactivate the myrosinase enzyme. On boiling, the Chinese cabbage was placed into the beaker, making sure it was submerged under water. Once the water containing the Chinese cabbage returned to a boil, it was allowed to continue for 3 more minutes. The beaker was then removed from heat and maintained at room temperature for $10 \mathrm{~min}$. The boiled cabbage was liquified with the water in a blender for $2 \mathrm{~min}$. The total volume of the homogenate was recorded and $100 \mathrm{~mL}$ of the homogeneous suspension was transferred to a $120-\mathrm{mL}$ plastic cup and frozen at $-30{ }^{\circ} \mathrm{C}$ until analyzed for glucosinolate content.

One day before analysis, frozen samples were thawed at room temperature and centrifuged at 13,000 $g_{\mathrm{n}}$ for $1 \mathrm{~h}(\mathrm{~J} 2-21 \mathrm{M} / \mathrm{E}$; Beckman Instruments, Fullerton, CA). The supernatant was transferred to a $20-\mathrm{mL}$ scintillation vial (03-337-2; Fisher Scientific Co., Pittsburgh, PA) and $5.6 \mathrm{~g}$ of ammonium sulfate was added per $10 \mathrm{~mL}$. Finally, the vials were stirred until the salt was completely dissolved and placed in a cold room $\left(5{ }^{\circ} \mathrm{C}\right)$ overnight to precipitate proteins that interfere with the high-performance liquid chromatography analysis. Before analysis, samples were retrieved from the cold room and filtered using $0.2-\mu \mathrm{m}$ syringe filters containing a surfactant-free cellulose acetate membrane (190-2520; Fisher Scientific Co.).

To analyze for gluconasturtiin concentration, an intact glucosinolate protocol was used (Lewke et al., 1996, modified by J.D. Cohen and L.Y. Wong, unpublished data).
Samples were analyzed using an Ultra Aqueous $\mathrm{C}_{18}$ analytical column $(4.6 \mathrm{~mm} \times 15 \mathrm{~cm}$, $5 \mu \mathrm{m}$; Restek USA, Bellefonte, PA) at $30^{\circ} \mathrm{C}$ with a flow rate of $1.0 \mathrm{~mL} \cdot \mathrm{min}^{-1}$. Solvent $A$ was $0.1 \mathrm{M}$ ammonium acetate and solvent $\mathrm{B}$ was $30 \%$ methanol with $0.1 \mathrm{M}$ ammonium acetate. The elution protocol was 0 to $6 \mathrm{~min}$, $100 \% \mathrm{~A} ; 6$ to $21 \mathrm{~min}$, a linear gradient to $70 \%$ $\mathrm{B}$; and 21 to $24 \mathrm{~min}$, a linear gradient to $100 \%$ B. The solvent was then held at $100 \% \mathrm{~B}$ for $11 \mathrm{~min}$. After the analytical sequence, a linear gradient to starting conditions ( 35 to $37 \mathrm{~min}$, linear gradient to $100 \% \mathrm{~A}$ ) followed by a 13 min postrun hold at $100 \%$ solvent A reconditioned the column for the next injection. Samples were analyzed in duplicate.

The external standards used to identify chromatogram peaks were prepared using a serial dilution starting from a stock of $30 \mu \mathrm{L}$ of $1.0 \mu \mathrm{g} \cdot \mu \mathrm{L}^{-1}$ of phenethyl glucosinolate potassium (gluconasturtiin) mixed with $120 \mu \mathrm{L}$ of distilled deionized water (ddwater) and then diluted in half three times.

Statistical analysis. Data from all experiments were analyzed using SAS (version 8.2; SAS Institute, Cary, NC). Testing for significance of main effects and interactions on all variables was conducted using analysis of variance. Data were analyzed using the PROC GLM procedure and DUNCANS for mean comparison separation.

\section{Results and Discussion}

Effect of cabbage looper density. Gluconasturtiin concentration significantly increased as a result of insect herbivory. However, the differences in gluconasturtiin concentration between the two larval treatments were not significant, regardless whether the plants were covered with netting as shown in Figure 1 (noncovered PPF: 400 umols $\cdot \mathrm{m}^{-2} \cdot \mathrm{s}^{-1}$ versus covered $P P F: 240$ $\left.\mu \mathrm{mols} \cdot \mathrm{m}^{-2} \cdot \mathrm{s}^{-1}\right)(P \leq 0.05)$. Rostás et al. (2002) found no effect of caging Chinese cabbage leaves with and without Phaedon cochleriae beetles on glucosinolate concentration. However, this is in contrast to what Charron and Sams (2004) observed in rapidcycling Brassica oleracea in which total glucosinolates decreased when $P P F$ was

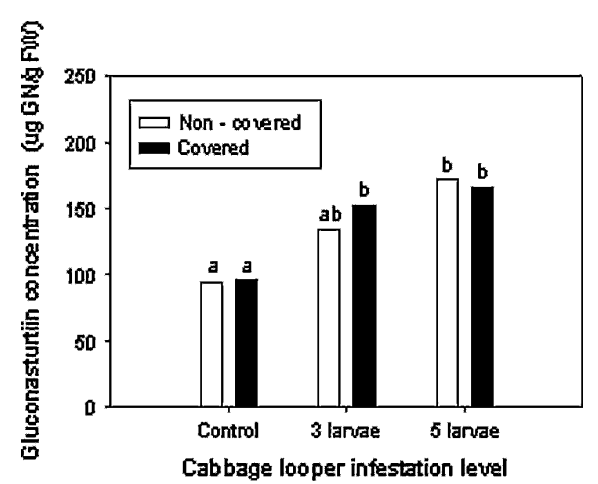

Fig. 1. Effect of cabbage looper larvae feeding on gluconasturtiin concentration in 'Green Rocket' Chinese cabbage after $10 \mathrm{~d}$ of feeding in $2002(P \leq 0.05)$. 
increased from 200 to $400 \mu \mathrm{mols} \cdot \mathrm{m}^{-2} \cdot \mathrm{s}^{-1}$. Because the difference in gluconasturtiin concentration between covered and noncovered plants was nonsignificant (Fig. 1), there was no significant influence of reduced light on gluconasturtiin production $(P \leq 0.05)$. However, it may be that differences in light levels were not large enough to influence gluconasturtiin concentration by affecting the metabolism of either the plant or insect.

An analysis of variance between the amount of leaf area removed by herbivory and the related gluconasturtiin concentration in remaining leaf tissue was conducted. Although a higher population of insects per plant resulted in lower leaf area, differences in gluconasturtiin were nonsignificant (data not shown). A regression analysis found a weak correlation between gluconasturtiin and leaf area removed $\left(\mathrm{y}=254.2-0.14 \mathrm{x} ; r^{2}=\right.$ $0.11 ; P \leq 0.05$ ), so leaf area loss alone does not appear to influence gluconasturtiin concentration in Chinese cabbage. Cipollini et al. (2003) also found no correlation between leaf area removed and total glucosinolate concentration in rape (Brassica napus) plants. This suggests that the effect of larval feeding (chewing) may be more important than any amount of leaf area removed. It is more likely that Chinese cabbage plants increased their gluconasturtiin concentration on experiencing damage incited by herbivory of the cabbage looper and not necessarily as a result of a reduction in leaf area (W.D. Hutchison, personal communication). Nielsen et al. (2001) suggested that Arabidopsis lines were induced to increase their sinalbin and total glucosinolate concentration regardless of the herbivore density of the specialist beetles Phyllotreta nemorum and P. cruciferae. Larval feeding could have caused a translocation of gluconasturtiin from wounded to nonwounded areas as suggested previously (Agrell et al., 2003; Bodnaryk, 1992). It is also possible that there is a more complex plant:insect interaction that may have accounted for the increase of gluconasturtiin. Kessler and Baldwin (2002) found that lytic enzymes in the oral secretions of Pieris brassicae larvae acted as elicitors of terpenoid volatiles from cabbage leaves, although there are no published data on a similar relationship between the saliva of cabbage looper and glucosinolates in crucifers.

Overall, we concluded that indeed, there was a significant increase of gluconasturtiin attributable to larval feeding compared with the noninfested controls, but no significant difference was noted between three and five larvae per plant.

Change in gluconasturtiin concentration after herbivory. The experiment was conducted twice in 2003. Ten days after cabbage looper feeding, gluconasturtiin concentration in Chinese cabbage significantly increased with five larvae per plant compared with control plants in both covered and noncovered plants as shown in Figure 2. There was no significant decrease in gluconasturtiin in the noncovered plants in both larval treatments at the last harvest (17 d after infesta- tion) compared with the second harvest ( $10 \mathrm{~d}$ after infestation). Once the stressor (larvae) was removed, gluconasturtiin levels were expected to return to normal, as suggested by Renwick (2002). It was also expected that gluconasturtiin levels would decline with plant age (Mewis et al., 2002). Agrell et al. (2003) has suggested that plants respond to injury within hours or days with relatively short lasting effects. However, this was not observed in our studies. Once gluconsaturtiin increased after the initial herbivory treat- ment, concentrations remained elevated $7 \mathrm{~d}$ after larval removal as shown in Figure 2. This may indicate that the increased gluconasturtiin concentration in plants subjected to herbivory results from accumulation of gluconasturtiin into a smaller pool of leaf tissue. This might suggest that there is a concentration effect and that the plant is compensating for tissue loss by increasing gluconasturtiin production per unit of leaf tissue.

To better evaluate the effect of larval feeding, we conducted a Duncan's multiple

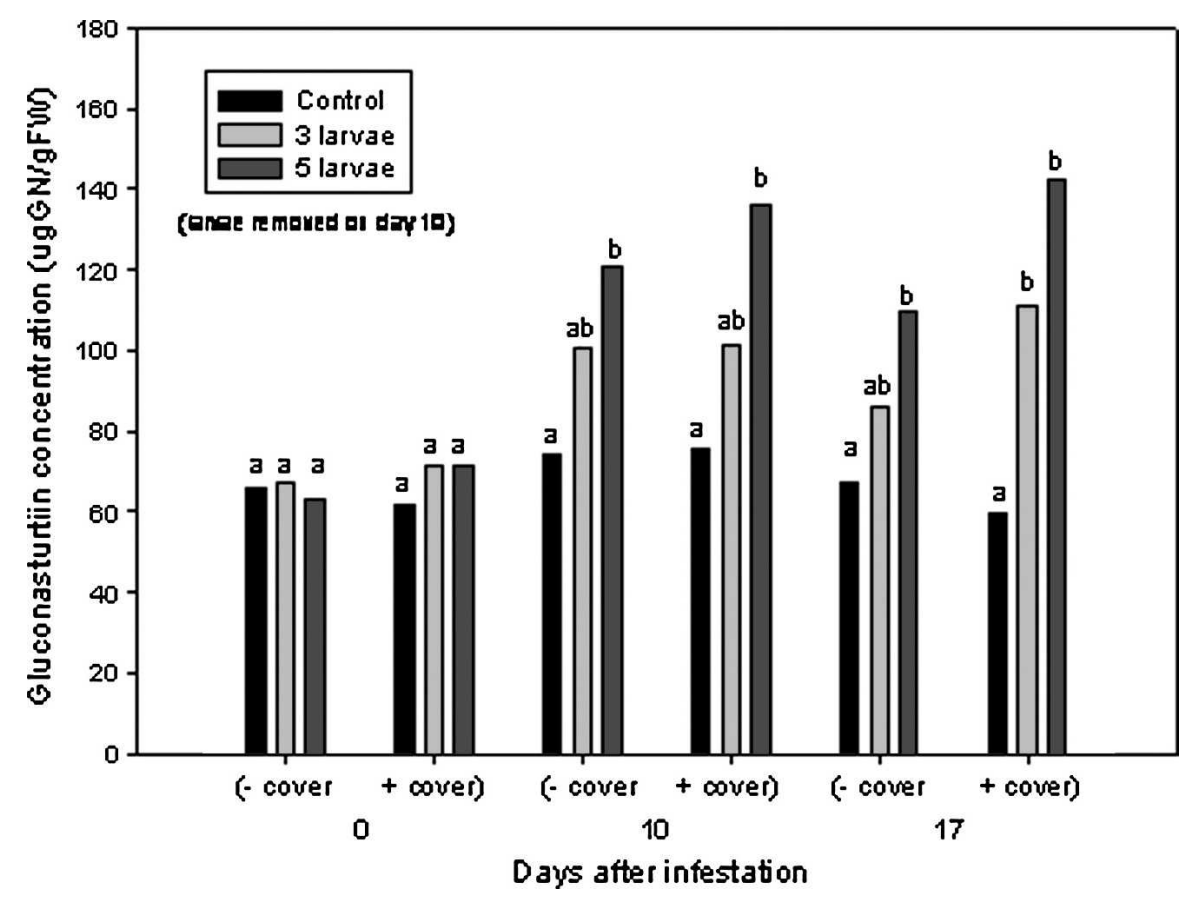

Fig. 2. Effect of immediate and postherbivory (17 d after infestation) cabbage looper larvae feeding on gluconasturtiin concentration in 'Green Rocket' Chinese cabbage in $2003(P \leq 0.05)$.

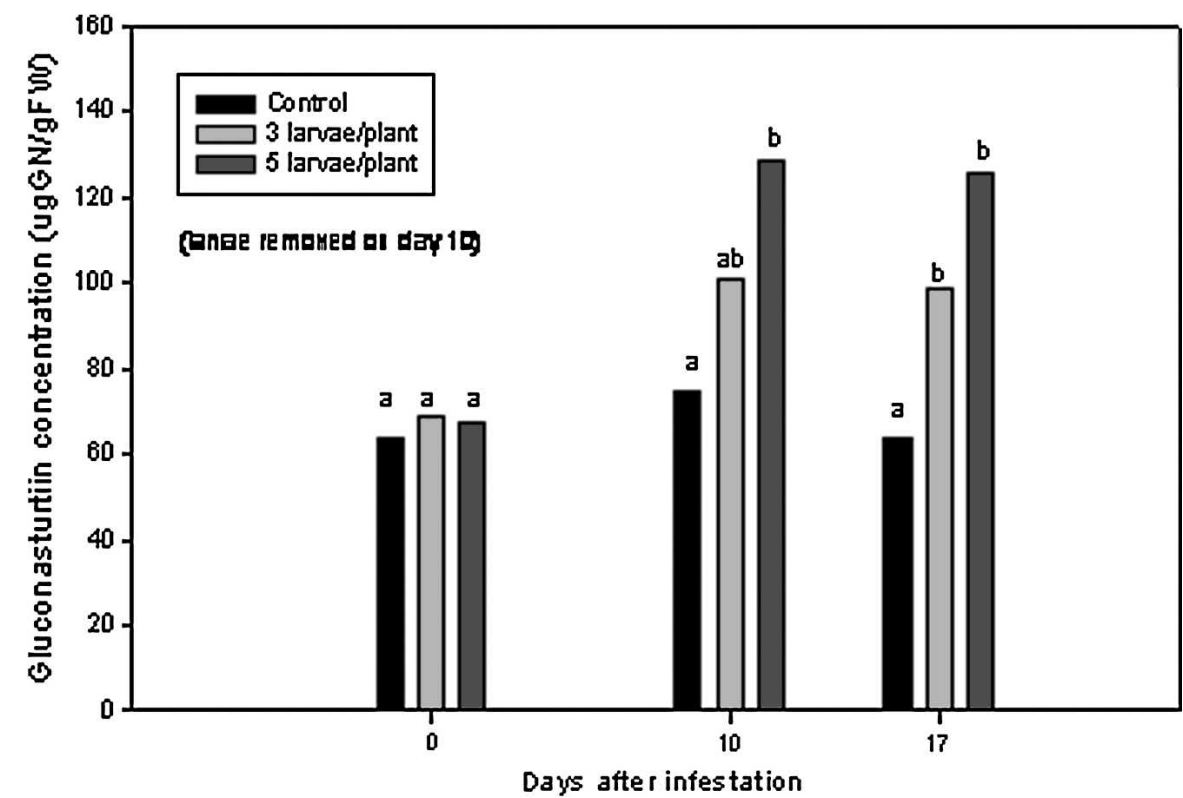

Fig. 3. Effect of immediate and postherbivory (17 d after infestation) cabbage looper larvae feeding on gluconasturtiin concentration in 'Green Rocket' Chinese cabbage, averaged over covering treatment, in $2003(P \leq 0.05)$. 
range mean separation test averaged over the covering (netting) treatments as shown in Figure 3. We confirmed that the treatment using five larvae per plant induced the plants to produce significantly more gluconasturtiin than the noninfested control plants $(P \leq 0.05)$ at the second and third harvests, but we found no significance between the two larval densities used.

These results confirmed that there was a significant increase in gluconasturtiin concentration when Chinese cabbage plants were damaged by cabbage looper feeding. It may be possible that the increase is because this aromatic glucosinolate is part of a plant defense mechanism against herbivory. Rostás et al. (2002) suggested that glucosinolates act against generalists (insects that feed on several vegetable species) and not against insects that feed primarily on crucifers (specialists). The cabbage looper might be considered a generalist, because it feeds on different species of vegetables. It may also be able to rapidly detoxify the compound or be stimulated to feed more aggressively by the increase in gluconasturtiin concentration. This action could force the plant into a rapid cycle of greater production of the compound, which was observed with five larvae per plant (Agrawal, 2000). It has also been reported that the potential repellent effect of gluconasturtiin is more effective in cabbage looper adults where they have been deterred from oviposition by high concentrations of glucosinolates (Kushad et al., 2004; Mewis et al., 2002).

Because nondamaged organs of the plant elevate their glucosinolate concentration when other organs within the same plant are damaged (Agrell et al., 2003; Bodnaryk, 1992), we analyzed changes in gluconasturtiin concentration in whole plants. This is important especially for potential marketing of specialty vegetables with enhanced health benefits. The damaged parts of a plant could be removed to improve the plant appearance while still benefiting from increased levels of glucosinolates.

Under our experimental conditions, leaf area and reduced light (noncovered, $P P F$ : $400 \mu \mathrm{mol} \cdot \mathrm{m}^{-2} \cdot \mathrm{s}^{-1}$ versus covered, $P P F: 240$ $\left.\mu \mathrm{mol} \cdot \mathrm{m}^{-2} \cdot \mathrm{s}^{-1}\right)$ conditions did not significantly affect gluconasturtiin concentration.

Public acceptance of vegetables with improved health benefits will depend to a large degree on a positive sensory experience. Elevated levels of glucosinolates in cruciferous vegetables are often associated with bitterness or pungency and could limit widespread embrace by the consumer. As fruits and vegetables are developed with the aim of increasing concentrations of naturally produced compounds with strong chemopreventive properties, management of compounds associated with a positive sensory reaction by consumers will be critical.

\section{Literature Cited}

Agrawal, A. 2000. Specificity of induced resistance in wild radish: Causes and consequences for two specialist and two generalist caterpillars. Oikos 89:493-500.

Agrell, J., W. Oleszek, A. Stochmal, M. Olsen, and P. Anderson. 2003. Herbivore-induced responses in alfalfa (Medicago sativa L.). J. Chem. Ecol. 29:303-320.

Andaloro, J. and A.M. Shelton. 1981. Insects of crucifers: Cabbage looper (Trichoplusia ni Hüebner) life cycle. Cornell Coop. Ext. Fact Sheet 102GFS751.00.

Bodnaryk, K.R.P. 1992. Effects of wounding on glucosinolates in the cotyledons of oilseed rape and mustard. Phytochemistry 31:2671-2677.

Charron, C.S. and C.E. Sams. 2004. Glucosinolate content and myrosinase activity in rapidcycling Brassica oleracea grown in a controlled environment. J. Amer. Soc. Hort. Sci. 129:321-330.

Chong, C. and B. Bible. 1974. Variation in thiocyanate content of radish plants during ontogeny. J. Amer. Soc. Hort. Sci. 99:159-162.

Cipollini, D., J. Busch, K. Stowe, E. Simms, and J. Bergelson. 2003. Genetic variation and relationships of constitutive and herbivoreinduced glucosinolates, trypsin inhibitors, and herbivore resistance in Brassica rapa. J. Chem. Ecol. 29:285-301.

Ju, H., B.B. Bible, and C. Chong. 1980. Variation of thiocyanate ion content in cauliflower and broccoli cultivars. J. Amer. Hort. Sci. 105:187-189.

Kessler, A. and I. Baldwin. 2002. Plant responses to insect herbivory: The emerging molecular analysis. Ann. Rev. Plant Biol. 53:299-328.
Kushad, M., R. Cloyd, and M. Babadoost. 2004. Distribution of glucosinolates in ornamental cabbage and kale cultivars. Scientia Hort. 101:215-221.

Lewke, A., A. Hanke, and W.H. Schnitzler. 1996. HPLC-analysis for intact glucosinolates of vegetable brassicaceae and their enzymatic detection by myrosinase degradation. Gartenbauwissenschaft 61:179-183.

Mewis, I., C. Ulrich, and W. Schnitzler. 2002. The role of glucosinolates and their hydrolysis products in oviposition and host-plant finding by cabbage web worm, Hellula undalis. Entomol. Exp. Appl. 105:129-139.

Nielsen, J., M. Hansen, N. Agerbirk, and B. Halkier. 2001. Responses of the flea beetles Phyllotreta nemorum and P. cruciferae to metabolically engineered Arabidopsis thaliana with an altered glucosinolate profile. Chemoecology 11:75-83.

Powolny, A., K. Takahashi, R. Hopkins, and G. Loo. 2003. Induction of GADD gene expression by phenethylisothiocyanate in human colon adenocarcinoma cells. J. Cell. Biochem. 90:1128-1139.

Renwick, J. 2002. The chemical world of crucivores: Lures, treats, and traps. Ent. Expt. Applic. 104:35-42.

Rosa, E.A., R.K. Heaney, G.R. Fenwick, and C.A.M. Portas. 1997. Glucosinolates in plants. Hort. Rev. 19:99-215.

Rostás, M., R. Bennett, and M. Hilker. 2002. Comparative physiological responses in Chinese cabbage induced by herbivory and fungal infection. Ent. Expt. Applic. 103:267-277.

Smith, T., R. Mithen, and I. Johnson. 2003. Effects of brassica vegetable juice on the induction of apoptosis and aberrant crypt foci in rat colonic mucosal crypts in vivo. Carcinogenesis 24 : 491-495.

Talalay, P. and J. Fahey. 2001. Phytochemicals from cruciferous plants protect against cancer by modulating carcinogen metabolism. J. Nutr. 131:3027S-3033S.

Wargovich, M.J. 2000. Anticancer properties of fruits and vegetables. HortScience 35:573-575.

Wattenberg, L.W. 1990. Inhibition of carcinogenesis by minor nutrient constituents of the diet. Proc. Nutr. Soc. 49:173-183.

Xiao, D., S. Srivastava, K. Lew, Y. Zeng, P. Hershberger, C. Johnson, D. Trump, and S. Singh. 2003. Allyl isothiocyanate, a constituent of cruciferous vegetables, inhibits proliferation of human prostate cancer cells by causing $\mathrm{G}(2) / \mathrm{M}$ arrest and inducing apoptosis. Carcinogenesis 24:891-897. 